\title{
"Psicologia Social do Trabalho": una intervención para el mundo laboral en América Latina
}

\author{
Hernán Camilo Pulido-Martínez ${ }^{1}$ \\ Pontificia Universidad Javeriana (Bogotá, Colômbia)
}

Reseña: Coutinho, M. C., Bernardo, M. H., \& Sato, L. (Orgs.) (2017). Psicologia Social do Trabalho. Petrópolis, RJ: Vozes. ISBN: 978-85-326-5549-3.

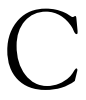
ontrario a ese tipo de textos de psicología organizacional que inundan las librerías, la propuesta que se presenta en el libro de Psicología Social do Trabalho resulta refrescante, interesante y muy retadora para aquellos psicólogos que buscan entender el mundo laboral en América Latina más allá de fórmulas preconcebidas. Sin lugar a duda, esta obra puede ser considerada como una intervención, tanto dentro de la disciplina psicológica, como en los ámbitos de trabajo. Numerosas son las razones por las cuales este texto que nos presentan académicos brasileros puede ser tomado como una intervención. Si se considera una definición amplia de intervención se puede afirmar que esta básicamente significa venir entre (Schvarstein, 1992). Con base en esta definición, la cuestión, entonces, que hay que poner sobre el tapete hace referencia a qué instancias, en cuáles temas y con quiénes el libro se ubica "entre".

\section{Introduce la categoría trabajo}

La estructura del libro por la que los autores optan señala su preocupación por partir en su reflexión de los problemas laborales propios de Brasil. Como muestra de esto, los investigadores se ocupan de examinar puntos centrales de la historia del trabajo, la cual le imprime características particulares al mundo laboral en ese país. Estas reflexiones no solamente se utilizan como contexto en donde ocurren los fenómenos psicológicos. Adicionalmente, ellas sirven para localizar la problemática y los argumentos que se van a desarrollar. Como muestras de las posibilidades que abre la introducción de la categoría trabajo para configurar una psicología social, los distintos capítulos la apropian de diversas maneras para mostrar cómo las especificidades locales llevan a consolidar propuestas alternativas y pertinentes a los problemas que aquejan a los trabajadores.

La introducción de la categoría trabajo resulta un "venir entre" pensar los problemas del trabajo y la trivialización de estos que se hace en la psicología hegemónica representada en las versiones industrial-organizacional que apartan al trabajo como tal de sus análisis. En las versiones hegemónicas de la psicología, el trabajo paradójicamente constituye solamente un telón de fondo contra el cual ocurren fenómenos psicológicos. Por el contrario, en la psicología social que se nos enseña, el trabajo es una parte constitutiva de los seres humanos y uno de los ámbitos centrales en donde se lleva a cabo la socialización. En este sentido, al introducir la categoría trabajo como eje articulador de los argumentos que se presentan en los diferentes capítulos, se abren una serie de perspectivas acerca de cómo se constituyen los seres humanos en los ámbitos laborales de

1 https://orcid.org/0000-0003-1071-2784 
acuerdo con la historia que antecede y con las formas que a lo largo del tiempo han consolidado una particular organización del trabajo.

Esto no quiere decir que el libro se convierta en una muestra exótica de la producción de conocimiento que solamente resulta pertinente para Brasil. Por el contrario, el hecho de presentar una manera de considerar cómo los fenómenos psicológicos tienen lugar gracias a las formas que asume el trabajo, proporciona las herramientas para que en otros países de la región, con situaciones y características similares, se utilice esta obra como una herramienta básica para pensar el mundo laboral local desde perspectivas inter y transdisciplinares.

\section{Delimita un campo}

También el libro hace una intervención en términos de demarcar un área del conocimiento en relación con los estudios que se interesan por el mundo laboral. La introducción del trabajo como fuente de los análisis trae consigo una articulación de conceptos que acuden para dar respuesta a las preguntas fuertes que la inclusión de esta categoría genera. El texto de Psicología Social del Trabajo representa entonces una intervención, ubicándose como campo alternativo caracterizado en otras por considerar las muy diversas formas que el trabajo toma en Brasil, y por qué no, en Latinoamérica.

El libro resulta ser una herramienta para psicólogos en formación y para profesionales, no solamente de esta disciplina, que estén interesados en lidiar con los problemas del trabajo de manera compleja. La delimitación del campo que se formula conlleva a que los autores reflexionen sobre las posibilidades y requerimientos que este tiene. Si la psicología convencional industrial organizacional se ubica al nivel de las relaciones entre la gerencia y los trabajadores, la psicología social del trabajo establece relaciones con los trabajadores directamente. Para establecer esta relación, y he aquí un requerimiento crucial de esta psicología, se requiere considerar como eje del campo las preguntas que formulen los trabajadores mismos, las organizaciones que los agrupan como los sindicatos, las entidades oficiales que promueven las políticas públicas y/o las ONGs interesadas en democratizar las relaciones que se establecen en la sociedad.

Ahora bien, en términos de la demarcación, esta obra hace un aporte al mostrar tanto, las condiciones de posibilidad que permitieron la emergencia de una psicología social del trabajo, como la cotidianidad misma del trabajo que contribuyó a constituirlo. Así, los autores señalan los momentos por los que atravesó Brasil en su historia en términos de las afectaciones que se produjeron en el mundo del trabajo, los antecedentes conceptuales y metodológicos que se dieron en las ciencias sociales y en la psicología misma, las muy variadas formas en como el trabajo se manifiesto en el diario vivir, así como también los movimientos sociales que labraron un terreno para se pudieran establecer las alianzas con los actores, no solamente pertenecientes a la academia, de modo tal que se lograron establecer unas manera de concebir y de llevar a cabo la producción de conocimiento.

\section{Reconoce un grupo académico}

Los autores, a través del libro, se muestran como grupo de investigadores que tienen intereses en común, posiciones éticas y políticas compartidas y muy distintos temas de investigación. Se describe a lo largo de la obra las experiencias y vicisitudes que el trabajo alternativo y crítico puede enfrentar, así como las coyunturas que han hecho posible que se configuren como grupo de investigadores. En este sentido, el libro se convierte en una especie de faro que puede permitir a otros grupos de investigadores, localizados en otros espacios geográficos, pensar las condiciones particulares que son necesarias para que la empresa crítica tenga posibilidades de desarrollo. El hecho de que la historia

\section{0}


tenga un lugar importante para pensar los problemas del trabajo lleva a que los autores reflexionen sobre su propia experiencia como productores de conocimiento y, por tanto, a que la plasmen como ejemplo del camino que tomaron para llegar a formular la propuesta del campo.

\section{Considera otras formas de trabajo}

El complejo mundo del trabajo de América Latina con su amplio rango de manifestaciones que cubre, por mencionar solamente algunas, el trabajo informal, los pequeños negocios, la supervivencia comunitaria, las fábricas recuperadas, las cooperativas de trabajo asociado, entre otras; no habían sido de interés para la psicología concentrada, fundamentalmente, en la forma empleo. Por el contrario, la psicología social del trabajo trae a la luz, esas formas invisibilizadas. Aún más, son estas formas las que conllevan el mayor interés de los autores. Esta es una operación con muchísima significancia debido a que la mayor parte de la fuerza laboral en América Latina se encuentra ubicada en este tipo de trabajos.

Por supuesto, no se trata de una cruzada contra el empleo, o la reivindicación romántica de esas formas de trabajo dejadas aparte. Más bien se trata de examinar las complejidades que se establecen en el mercado laboral y los modos en los cuales las diferentes formas de trabajar se complementan para sostener la economía, precarizar el trabajo y establecer relaciones entre lo rural y lo urbano, entre lo formal y lo informal, entre el trabajo regulado y el que no lo está. En estas condiciones, al poner de presente formas de laborar desconocidas, invisibilizadas o no suficientemente exploradas por instancias, tales como la psicología industrial-organizacional, la relación entre el empleo y los otros medios de trabajo, permite pensar no solo las condiciones del primero, sino de todas otras aquellas múltiples formas que están paralelamente teniendo lugar en el variado espectro de las modalidades de trabajo presentes en la contemporaneidad.

\section{Promueve un método}

La psicología social del trabajo propuesta en el libro hace una apuesta en donde teoría y práctica, investigación e intervención, no se consideran como momentos independientes o separados dentro del proceso de conocer. La teoría ilumina la práctica y la practica a la teoría. Enmarcados en la destitución de esta separación, se examinan los problemas concretos que tienen los trabajadores en su día a día. Con el privilegio del punto de vista de los trabajadores se contribuye a constituir explicaciones sobre los fenómenos laborales, y sobre la constitución de los trabajadores mismos, que no tienen como característica central el cuño individualista, responsabilizante y culpabilizante de aquellos que trabajan.

Es decir, los resultados de los ejercicios de investigación-intervención se alejan de las visiones hegemónicas que se concentran en investigar para lograr la eficiencia y la productividad, tal como ocurre en la psicología industrial-organizacional; en su lugar, con una visión holista se investiga e interviene no para los trabajadores, sino con ellos. En este modo asumido de construir el conocimiento que se presenta en esta contra-psicología, se busca naturalmente construir unas relaciones más equitativas entre investigadores e investigados en donde no se observe a unos y se prescriban recetas con el conocimiento que se obtuvo de ellos. La construcción del conocimiento se lleva a cabo como una cuestión negociada y colaborativa entre académicos y trabajadores, así como de otros actores que puedan estar participando, regulando o interviniendo el mundo laboral. Si bien, la democratización de las relaciones entre los participantes en una investigación no es novedosa para las ciencias sociales, ni para otras ramas de la psicología, si resulta relativamente nueva para las ramas que se ocupan del trabajo colonizadas por visiones positivistas y leves del conocimiento psicológico. 
También, en términos de método es importante señalar que los autores consideran que todas las situaciones de trabajo son complejas. ¿A qué hacen referencia cuando afirman que las situaciones de trabajo son complejas? Primero, que no se revelan las aristas de un problema laboral en toda su dimensión con observaciones indirectas y ocasionales como suele ocurrir en las versiones en donde, por ejemplo, la medición del clima organizacional o de la cultura laboral son fundamentales. En su lugar, la exploración de la experiencia de los trabajadores aporta una perspectiva amplia de cuáles son y han sido los problemas del trabajo en ámbitos específicos. Esta perspectiva hace que los autores del libro hayan escogido la experiencia particular del trabajo como foco que puede ser estudiado a partir de metodologías participativas de corte cualitativo y de larga permanencia en el campo. La etnografía, la historia oral, la recuperación de las trayectorias laborales, nada más por citar algunas formas de proceder, pueden resultar coherentes con este tipo de propuesta de investigaciónintervención en donde se establecen los problemas cotidianos de los trabajadores para enfrentar sus causas generadoras, con el objetivo de que los resultados sean útiles para los mismos trabajadores.

Falta anotar que los autores señalan que, adicionalmente, cuando investigación e intervención no se separan, y aquí otra característica que ubica a la psicología social del trabajo en el campo de las contra-psicologías, se desdibuja o se borra otro de los límites que la psicología tradicional ha establecido para sí, es decir, se rompe la división entre psicología pura o teórica y psicológica aplicada.

\section{Requiere de la inter y la transdisciplinariedad}

Asimismo, el libro se le puede considerar como una intervención en relación con la manera en que propone sus vínculos con otros campos afines para entender los problemas del trabajo. La propuesta de una psicología social, que se encargue de lo laboral en situación, emerge en medio de las preguntas por la interdisciplinariedad que contemporáneamente ronda en las facultades de psicología. En la psicología social del trabajo que los autores configuran, la interdisciplinariedad no es un deseo por alcanzar, es un hecho que se requiere para poder resolver las preguntas que se le formulan. La experiencia particular de cada investigador y del grupo al que pertenece, así como las vivencias de los trabajadores con los cuales se comparte la indagación, acarrea que los problemas del trabajo se definan en un contexto amplio, en un panorama extenso, en el cual se encuentra el juego entre lo particular de un caso que ocurre en el mundo del trabajo y el contexto histórico, político, social económico y cultural en el que este tiene lugar.

Como se recomienda para aquellos interesados en hacer unos trabajos similares a los que se presentan, un equipo constituido por profesionales de diferentes áreas del conocimiento resulta ideal. A este respecto, vale anotar que en las raíces mismas de la psicología social del trabajo se encontraban campos que provenían de vertientes múltiples, que al mezclarse resultaron ser más que una adición de perspectivas procedentes de disciplinas independientes. La medicina social y el operaísmo italiano estuvieron llamados a la hora de sentar unas bases conceptuales para la intervención de los problemas del trabajo que esta contra-psicología quiere confrontar.

Las categorías elegidas por esta contra-psicología hacen gala de la necesidad de considerar perspectivas inter y transdisciplinares. Prácticas cotidianas, sentidos construidos, conflicto entre capital y el trabajo, así como la construcción de la identidad considerados como focos de estudio requieren de relaciones entre campos instituidos del saber, así como la creación de miradas transdisciplinares. Cada uno de por sí, y la relación entre los temas señalados, abre la necesidad de una serie de diálogos entre perspectivas que permitan dar cuenta de ellos. Y es que la tarea no es fácil, pues se trata de conjugar de modo inter o transdisciplinar a las ciencias sociales, a la filosofía, a la ergonomía, a la medicina y a la psicología en un dialogo intenso acerca de las situaciones cotidianas de trabajo concretas, a las cuales se les considera como el punto en donde se encuentran, 
los contextos macrosociales, con la formación de la subjetividad a través de la vivencia que se experimenta en el trabajo.

Aquí, sin embargo, como señalan los autores, hay una cierta debilidad del conocimiento que se presenta, puesto que aún falta más elaboración y posiblemente articulación entre los campos que pueden ser utilizados para repercutir en la transformación del trabajo. Por ejemplo, una psicología de corte aún más histórico podría estar en mora de formularse, con esta se podría, como se indica, hacer inferencias sobre la relación entre la constitución del sujeto y los cambios en el capitalismo en las condiciones particulares y múltiples que tiene el mundo del trabajo.

\section{Una posición política comprometida}

En este punto la intervención que se presenta en el libro, por una parte, está relacionada con venir entre aquellas perspectivas de la psicología convencional que asumen que se puede hacer una serie de mejoramientos constantes que no cuestionan el orden del trabajo que genera problemas de inequidad, exclusión, precarización y desempleo, y por otra parte, entre aquellas que asumen perspectivas cualitativas que producen conocimiento desde los trabajadores para conocerlos en mayor profundidad y después realizar acciones que mejoren el desempeño administrativo. Irrumpe, entonces, la psicología social del trabajo para formular una perspectiva que supone que el conocimiento se debe producir no desde y para el otro, sino en este caso con los trabajadores.

En esta línea, es imposible pensar en una neutralidad, pues la reflexión parte de considerar que los sitios de trabajos son ante todo espacios políticos. Por esta razón, como los autores manifiestan, es crucial partir de la vivencia de la cotidianidad laboral para examinar las contradicciones del sistema que se hacen patentes en el quehacer diario del trabajo. En este sentido, se produce conocimiento no para instruir al trabajador pues él es experto en las contradicciones que enfrenta; mejor se investiga e interviene, para la molestia de otras ramas de la psicología, para la promoción de acciones afirmativas en donde los trabajadores y los investigadores son actores políticos capaces de influir y cambiar los ámbitos laborales.

El proceso de investigación e intervención abre un espacio para que el trabajador interactúe con lo que será su resultado. Entonces, hay que resaltar que quizá ahí radique la posible efectividad de este, ya que la transformación de las condiciones de trabajo estará directamente vinculada con aquellos a quienes afecta. Habría que agregar que esta posición para conducir la investigaciónintervención también debería demandar una reflexión del investigador como trabajador que, de cierta forma en la misma condición del investigado, comprende su cotidianidad en el contacto con otras cotidianidades de trabajo en algún grado distintas. En su condición de investigador/ trabajador, quizá uno de los principales aportes está representado en su competencia para establecer condiciones físicas del trabajo en la que tiene lugar la organización del trabajo, su capacidad para hacer análisis críticos de las relaciones sindicales patronales, sus posibilidades para hacer recuentos de la historia del trabajo en el contexto bajo estudio, así como para recolectar información acerca de las condiciones y relaciones del trabajo.

Las actividades de la producción de conocimiento que se llevan a cabo, además, son una actividad política al menos en los siguientes aspectos. En la medida en que se describen y analizan las condiciones laborales, los diferentes entes académicos, gremiales y gubernamentales pueden hacer públicas dichas condiciones, así se ponen en la agenda social condiciones, por ejemplo, precarias, dañinas, inequitativas, para su posible transformación a través de diferentes estrategias. Igualmente, los resultados de las investigaciones construidas con los trabajadores deben ser viables para que estos las puedan utilizar como "municiones" para cambiar el trabajo. Adicionalmente, el investigador tiene un papel político explícito como "activista" en la medida en que su rol y sus 
prácticas están reconocidamente comprometidas con los trabajadores. Por tanto, debe apuntar a llevar a cabo acciones afirmativas conjuntas que replanteen las tensiones presentes entre capital, trabajo y estado, para de este modo construir una reflexión sobre las posibilidades mismas de transformación social necesaria para la construcción de nuevas relaciones de poder en el trabajo.

Falta considerar que en muchas perspectivas críticas propuestas en relación con la psicología y el trabajo se asume un papel diagnóstico que deja un sabor de pesimismo e inmovilidad. Ha sido muy valiosa la perspectiva crítica para develar las implicaciones que tiene la psicología cuando se aplica al mundo del trabajo. Empero, contrario a lo que ocurre en algunas áreas de la disciplina, la crítica no ha conseguido cuestionar fuertemente ni el conocimiento, ni las prácticas que se llevan a cabo en los ámbitos laborales; la muestra de ello es que no se ha logrado hacer algún tipo de desgaste de la psicología hegemónica presente en los ámbitos laborales. La psicología social del trabajo que está contenida en esta obra es una intervención, de por sí ya significa una movilización que no vuelve sobre, o mejor, no se detiene en la cuestión puntual de proponer una psicología mejorada. El libro hace parte de un programa en donde intervención e investigación no se separan, sino que son constitutivos el uno del otro. No se puede entonces separar este texto del programa que desde hace décadas llevan a cabo los académicos que hacen parte de esta obra.

A manera de cierre, solamente cabe señalar una pregunta adicional, si a lo largo del libro se está desenvolviendo un tipo de conocimiento distinto, complejo, político y transformador, ideberá querérsele mantener dentro de los límites de la disciplina psicológica?, quizá sea el tiempo de apartarse de una vez y por todas de las restricciones que impone mantenerse dentro del complejo psi.

\section{Referencias}

Parker, I. (2007). Revolution in Psychology: alienation to emancipation. Londres: Pluto Press.

Schvarstein, L. (1992). Psicologia Social de las Organizaciones: nuevos enfoques. Buenos Aires: Paidos.

Endereço para correspondência

cpulido@javeriana.edu.co
Recebido em: 21/04/2020

Aprovado em: 05/05/2020 